\title{
PAIRED AND CLUSTERED QUANTUM HALL STATES
}

\author{
K. Schoutens, E. Ardonne and F.J.M. van Lankvelt \\ Institute for Theoretical Physics, Valckenierstraat 65 \\ 1018 XE Amsterdam, The Netherlands
}

\begin{abstract}
We briefly summarize properties of quantum Hall states with a pairing or clustering property. Their study employs a fundamental connection with parafermionic Conformal Field Theories. We report on closed form expressions for the many-body wave functions and on multiplicities of the fundamental quasi-hole excitations. ${ }^{1}$
\end{abstract}

Keywords: quantum Hall effect, pairing, CFT, parafermions, non-abelian statistics

\section{Introduction: new states of matter in the quantum Hall and BEC arenas}

The fractional quantum Hall effect has unveiled states of matter that can be characterized as incompressible quantum fluids with topological order. Such states are formed in a two-dimensional electron gas, at very low temperature and in the presence of a strong perpendicular magnetic field. It has been recognized early on $[17,7]$ that the excitations over fractional quantum Hall states obey fractional braid statistics: a configuration of $N$ quasi-holes over a fractional quantum Hall ground state forms a one-dimensional representation of the braid group $B_{N}$, where the braiding of two quasi-holes is typically represented by $e^{i \alpha \pi}$, with $\alpha$ a rational but non-integer number. The requirement that particle states have to represent the braid group rather than the permutation group is special for two dimensions: the braid group is the fundamental group of the configuration space of identical particles only in two dimensions. On general grounds it is known that, for two-dimensional quantum systems, higher dimensional representations of the braid group $B_{N}$ are allowed ${ }^{1}$ Contribution to the proceedings of the NATO Advanced Research Workshop "Statistical
Field Theories" Como (Italy), June 18-23 2001 
(see [13] for an early reference). In such a situation, the braiding of particles is represented by matrices, and since matrices in general do not commute, this leads to the notion of non-abelian statistics.

It is now believed that the 'non-abelian statistics scenario' is realized in novel types of quantum Hall states, which are characterized by a pairing or clustering of electrons under quantum Hall conditions. There exists concrete experimental [32] and numerical [21] evidence that the simplest of these states, the 'pfaffian state' proposed by Moore and Read [22], exists in nature, as the state of matter underlying the quantum Hall effect at filling fraction $\nu=\frac{5}{2}$. At present, this example stands as the lone confirmed 'sighting' of a non-abelian quantum Hall state. One expects that paired and clustered quantum Hall states can be realized in multi-layer quantum Hall systems with sufficiently strong interlayer tunneling, or in situations where the spin of the electrons is not fully polarized.

It has recently become clear that quantum Hall states with clustering correlations are relevant for a very different class of physical systems, namely Bose Einstein condensates (BEC) of cold atoms in a rotating trap. In a regime where the rotation frequency $\Omega$ is of the order of the frequency $\omega$ set by the confining potential, and where the healing length $\xi$ is very large, a rotating BEC becomes formally analogous to a quantum Hall system of bosonic particles. In numerical studies, the quantum liquid that is formed in a rotating BEC at vorticity $\nu=N_{\text {boson }} / N_{\text {vortex }}=$ $k / 2$, has been identified with the Read-Rezayi quantum Hall state with order- $k$ clustering [10].

In this paper we briefly review the properties of a variety of paired and clustered quantum Hall states, with particular attention for states formed by spinful electrons. We present closed form expressions for ground state wave functions and discuss the multiplicities and statistics properties of quasi-hole excitations. Throughout our presentation, we stress the role of parafermionic conformal field theory as a crucial tool in the theoretical description.

\section{The fractional quantum Hall effect}

The discovery of the fractional quantum Hall (fqH) effect [28] was truly remarkable and unanticipated. At fractional filling fraction $\nu=1 / 3$ a quantum Hall $(\mathrm{qH})$ plateau was observed. The filling fraction is defined as the ratio of the number of electrons and the number of available states in the lowest Landau level: $\nu=N / N_{\phi}$, where $N_{\phi}$ is the number of flux quanta piercing the sample, and $N$ the number of electrons. Soon after the discovery, Laughlin made a fundamental break-through by proposing 
his by now famous wave functions, which describe the $\mathrm{qH}$ effect at filling fraction $\nu=\frac{1}{m}$, where $m$ is an odd integer [20]

$$
\widetilde{\Psi}_{\mathrm{L}}^{m}\left(z_{1}, \ldots, z_{N}\right)=\prod_{i<j}\left(z_{i}-z_{j}\right)^{m}
$$

Here and below we display reduced $\mathrm{qH}$ wave functions $\widetilde{\Psi}(z)$, which are related to the actual wave functions $\Psi(z)$ via $\Psi(z)=\widetilde{\Psi}(z) \exp \left(-\sum_{i} \frac{\left|z_{i}\right|^{2}}{4 l^{2}}\right)$ with $l=\sqrt{\frac{\hbar c}{e B}}$ the magnetic length.

Although the $\mathrm{qH}$ effect occurs at relatively high magnetic fields, it was soon realized that the electron spin can indeed play an important role. The spin-polarized Laughlin states were generalized by Halperin, who proposed a set of spin-singlet wave functions [18]

$$
\begin{aligned}
& \widetilde{\Psi}_{\mathrm{H}}^{m+1, m+1, m}\left(z_{1}^{\uparrow}, \ldots, z_{N}^{\uparrow} ; z_{1}^{\downarrow}, \ldots, z_{N}^{\downarrow}\right)= \\
& \prod_{i<j}\left(z_{i}^{\uparrow}-z_{j}^{\uparrow}\right)^{m+1} \prod_{i<j}\left(z_{i}^{\downarrow}-z_{j}^{\downarrow}\right)^{m+1} \prod_{i, j}\left(z_{i}^{\uparrow}-z_{j}^{\downarrow}\right)^{m},
\end{aligned}
$$

where $z_{i}^{\uparrow}$ and $z_{j}^{\downarrow}$ are the coordinates of the spin-up and spin-down electrons, respectively. The state Eq. (2) has filling fraction $\nu=2 /(2 m+1)$.

\subsection{The qH effect-CFT connection}

Bulk connection. Following Moore and Read [22] one observes that it is natural to view (lowest Landau level) $\mathrm{qH}$ wave functions as conformal blocks of electron-type operators in a suitable chiral conformal field theory $(\mathrm{CFT})$ in $2+0$ dimensions. This point of view is related to the fundamental role of Chern-Simons field theories for $\mathrm{qH}$ systems (compare with [33], where an explicit link between Chern-Simons theory and CFT is established).

As an example, the Laughlin ground state wave function (1) is obtained as

$$
\widetilde{\Psi}_{\mathrm{L}}^{m}=\lim _{z_{\infty} \rightarrow \infty}\left(z_{\infty}\right)^{m N^{2}}\left\langle V_{e}\left(z_{1}\right) \ldots V_{e}\left(z_{N}\right): e^{-i \sqrt{m} N \varphi}\left(z_{\infty}\right):\right\rangle
$$

with $V_{e}(z)=: \exp (i \sqrt{m} \varphi)$ : a chiral vertex operator in the $c=1$ chiral CFT describing a single scalar field $\varphi$ compactified on a radius $R^{2}=m$.

Edge connection. While bulk excitations over a $\mathrm{qH}$ fluid are gapped, one expects gapless excitations at the edge of a sample. Following Wen [29], one observes that the edge excitations are described by a chiral 
Luttinger Liquid or chiral CFT in $1+1$ dimensions. In the example of the $\nu=\frac{1}{m}$ Laughlin states, one again has the scalar field theory at $R^{2}=m$. The neutral operator $\rho=i \sqrt{m} \partial \varphi$ is associated with edge density waves, while vertex operators of type $V^{q}(z)=: \exp (i q \sqrt{m} \varphi)$ : represent charged edge excitations, the charge being equal to qe with $-e$ the charge of the electron and $\frac{e}{m}$ the charge of the fundamental quasi-holes.

\subsection{Fractional statistics in the fqH effect}

In the case of an abelian $\mathrm{qH}$ state, changing the magnetic field by one flux quantum $\Phi_{0}=\frac{h}{e}$ results in the creation of a quasi-hole (or particle, depending on the sign of the change). These quasi-holes can have remarkable properties, such as a fractional charge. Also, the quasiholes over the Laughlin $\mathrm{fqH}$ states are anyons, i.e. they realize fractional braid statistics $[17,7]$. The fundamental phase for the braiding of two such excitations is given by $e^{i \frac{\pi}{m}}$.

Closely related to this are the fractional exclusion statistics of these same excitations $[16,19]$. Focusing on edge excitations, one can show that the gapless, charged edge excitations of an abelian $\mathrm{qH}$ state satisfy a form of exclusion statistics closely related to that of Haldane [16]. A particularly natural choice of basis for the edge excitations employs edge electrons and quasi-holes as the fundamental quanta [11]. In this basis, the exclusion statistics parameter matrix is diagonal with self-exclusion parameters equal to $m$ (for the edge electrons) and $\frac{1}{m}$ (for the edge quasi-holes).

For general abelian $\mathrm{qH}$ states, one may argue [1] that the statistics matrix $G$ of edge excitations (in a specific basis) is of the form

$$
\mathbf{G}=\mathbf{K}_{e} \oplus \mathbf{K}_{e}^{-1}
$$

where $\mathbf{K}_{e}$ is the so-called $K$-matrix that characterizes the topological order of the $\mathrm{qH}$ state (see for instance [30]).

\section{3. $\quad$ Paired and clustered $\mathrm{qH}$ states}

Prompted by the observation of a $\mathrm{qH}$ effect at filling fraction $\nu=\frac{5}{2}$ [32] a number of novel $\mathrm{qH}$ states have been proposed. Among these is the Moore-Read (MR) state or pfaffian state, which is characterized by a $p$-wave pairing of the electrons $[22,14]$. A generalization, where the pairing is replaced by a clustering of order $k$ was proposed by Read and Rezayi (RR) [25]. In [5] two of the present authors made a further generalization to a class of spin-singlet $\mathrm{qH}$ states also characterized by a clustering into $k$-plets of electrons. 


\subsection{Spin-polarized states}

The wave function of the (spin polarized) MR state is given by

$$
\widetilde{\Psi}_{\mathrm{MR}}\left(z_{i}\right)=\operatorname{Pf}\left(\frac{1}{z_{i}-z_{j}}\right) \prod_{i<j}\left(z_{i}-z_{j}\right)^{M+1}
$$

where $\operatorname{Pf}\left(M_{i, j}\right)=\frac{1}{2^{N / 2}(N / 2) !} \sum_{\sigma} \operatorname{sgn}(\sigma) \prod_{r=1}^{N / 2} M_{\sigma(2 r-1), \sigma(2 r)}$ is the pfaffian of an antisymmetric matrix. For the wave function to be antisymmetric (we are describing electrons) $M$ needs to be an odd integer, which implies an even-denominator filling fraction $\nu=\frac{1}{M+1}$.

The MR wave function can be viewed as a correlator in a $c=3 / 2$ CFT, consisting of a free scalar field and a Majorana fermion. The electron operator becomes $\psi(z): \exp \left(i \sqrt{M+1} \varphi_{c}\right):(z)$. The correlator of $N$ electron operators (and a suitable background charge) splits into a product of vertex operators, giving the Laughlin part of the wave function, and a product of fermion fields, which gives the pfaffian factor.

Upon generalizing the Majorana fermion to the $\mathbb{Z}_{k}$ parafermions [34] associated to the coset $\frac{\widehat{\mathrm{su}}(2)_{k}}{\widehat{\mathrm{u}}(1)}$, one obtains the clustered states of [25]. Their wave functions are constructed in the same way as the MR wave function, with explicit parafermion factors brought in by the electron operator. The result is a state in which the electrons form clusters of order $k$ rather than pairs. The filling fraction takes the form $\nu=\frac{k}{k M+2}$, with $M$ an odd integer. Note that for $k=1$ the Laughlin states (with $m=M+2$ ) are recovered, while $k=2$ gives the MR states.

In ref. [9], Cappelli et al. presented a particularly simple construction for $k$-clustered qH states, and they showed that the resulting wave functions are equivalent to the ones originally obtained by Read and Rezayi [25]. In this construction, one starts with an abelian state of $k$ types of discernible electrons, with the same total filling fraction as the clustered states. For the RR states, this abelian state takes the form [9]

$$
\widetilde{\Psi}_{\mathrm{ab}}=\prod_{a=1}^{k} \prod_{i<j}\left(z_{i}^{a}-z_{j}^{a}\right)^{2},
$$

where $a$ labels the different types of electrons. In the construction, we will assume that $M=0$; the wave function for $M \neq 0$ are obtained by multiplying the final result with the Laughlin factor $\prod_{i<j}\left(z_{i}-z_{j}\right)^{M}$. Note that the wave function (6) can be thought of as $k$ copies of the clustered state with parameters $k=1, M=0$ (which is a bosonic Laughlin state at filling fraction $\nu=\frac{1}{2}$ ). The clustered states are obtained by effectively 
making the electrons indiscernible, which is achieved by symmetrizing the expression (6) over all the electrons. The wave function for the RR states can now be written in the following form

$$
\widetilde{\Psi}_{\mathrm{RR}}=\mathcal{S}\left[\prod_{a=1}^{k} \prod_{i<j}\left(z_{i}^{a}-z_{j}^{a}\right)^{2}\right] \prod_{i<j}\left(z_{i}-z_{j}\right)^{M}
$$

where it is assumed that after the symmetrization procedure all the electrons are labeled by a single index.

The clustering property of the wave function (7) for $M=0$ is inherited from the abelian state (6). The clustering property of order $k$ means that the wave function does not vanish if up to $k$ particles are brought to the same position; however, if $k+1$ particles are at the same position, the wave function does vanish. To see this, note that $k$ electrons all of different type are brought to the same location, the wave function (6) does not vanish. The symmetrization makes sure that this property holds for any $k$ electrons of the clustered state (7) (with $M=0$ ).

\subsection{Spin-singlet states}

The clustered states discussed in the previous section do not include a spin degree of freedom. As was shown in [5] and [2], the clustering property can be extended to spin-singlet states of spinful electrons, leading to what have been called 'non-abelian spin singlet' (NASS) states. As stated before, the Halperin states are spin-singlet analogues of the Laughlin states. In the same way, the NASS states of [5] are spin-singlet analogues of the RR states. To construct their wave functions, two boson fields are needed (for charge and spin) in addition to the 'higher rank' parafermions associated to $\frac{\widehat{\mathrm{su}}(3)_{k}}{\widehat{[\mathrm{u}(1)]^{2}}}[12]$. The NASS states have fill-

ing fraction $\nu=\frac{2 k}{2 k M+3}$ with $M$ an odd integer. For $k=1$ the Halperin states (2), with $m=M+1$, are recovered. Explicit expressions for the NASS wave functions of [5] can be found in [4]. The form of the wave functions presented there is similar to the explicit $R R$ wave functions in [25]. Alternatively, a formulation similar to the construction for the RR states presented above is also possible. We again start with $k$ copies of the $k=1, M=0$ states, which are, in this case, the Halperin states with labels $(2,2,1)$. Again, this is a bosonic state. To obtain the fermionic states, one has to multiply in the end with an overall Laughlin factor $\prod_{i<j}\left(z_{i}-z_{j}\right)^{M}$ with $M$ odd. The abelian state from which the clustered 
spin-singlet states can be obtained takes the form

$$
\widetilde{\Psi}_{\mathrm{ab}}=\prod_{a=1}^{k}\left(\prod_{i<j}\left(z_{i}^{\uparrow, a}-z_{j}^{\uparrow, a}\right)^{2} \prod_{i<j}\left(z_{i}^{\downarrow, a}-z_{j}^{\downarrow, a}\right)^{2} \prod_{i, j}\left(z_{i}^{\uparrow, a}-z_{j}^{\downarrow, a}\right)\right) .
$$

Again, we have $k$ types of electrons, but now also the spin is taken into account. It is not so hard to find the filling fraction of this abelian state, which is given by $\nu=\frac{2 k}{3}$, which is indeed the filling fraction of the NASS states with $M=0$. To obtain the clustered state, one has to symmetrize the wave function (8). However, because we are dealing with spin-singlet states, the symmetrization procedure is more involved than for the RR states. The conditions which are to be satisfied by the spatial part of the wave function in order be a spin-singlet are the Fock cyclic condition, and the conditions that the wave wave function be symmetric in the spin-up and spin-down coordinates separately. In general, this can be achieved by the following (generalized) symmetrization procedure (see [4] for more details). First antisymmetrize in $z_{1}^{\uparrow}, z_{1}^{\downarrow}$, followed by antisymmetrization in $z_{2}^{\uparrow}, z_{2}^{\downarrow}$, etc. After the antisymmetrization of $z_{N / 2}^{\uparrow}, z_{N / 2}^{\downarrow}$, the wave function has to be symmetrized over all the spin-up coordinates, and finally, over the spin-down coordinates. This gives rise to clustered spin-singlet wave functions. Though we do not have a proof, we believe that via this procedure, we obtain wave functions which are equivalent to the ones described in [4]. For the case at hand, the antisymmetrization steps as described above do not seem to be necessary (as was the case for the explicit formulation in [4]). Thus we arrive at the following form for the wave functions of the clustered spin-singlet states

$$
\begin{aligned}
& \widetilde{\Psi}_{\mathrm{NASS}}= \\
& \mathcal{S}_{z^{\uparrow}, z^{\downarrow}}\left[\prod_{a=1}^{k}\left(\prod_{i<j}\left(z_{i}^{\uparrow, a}-z_{j}^{\uparrow, a}\right)^{2} \prod_{i<j}\left(z_{i}^{\downarrow, a}-z_{j}^{\downarrow, a}\right)^{2} \prod_{i, j}\left(z_{i}^{\uparrow, a}-z_{j}^{\downarrow, a}\right)\right)\right] \\
& \times \prod_{i<j}\left(x_{i}-x_{j}\right)^{M},
\end{aligned}
$$

where $x_{i}$ stands for either $z_{i}^{\uparrow}$ or $z_{i}^{\downarrow}$. $\mathcal{S}_{z \uparrow, z \downarrow}$ denotes symmetrization over the spin-up and spin-down particles. The clustering property of these spin-singlet wave functions can again easily be read off from this formulation. For more details, see [4].

Recently, another type of clustered spin-singlet $\mathrm{qH}$ states was proposed [2]. These states are based on parafermions corresponding to 
another rank 2 affine Lie algebra, namely the $\frac{\widehat{\mathrm{so}}(5)_{k}}{\widehat{[\mathrm{u}(1)]^{2}}}$ parafermions. Also, two chiral boson fields for the charge and spin degrees of freedom are present. At level $k=1$, the only parafermion field present in this theory is the Majorana fermion, which also appeared in the theory for the MR state. It is thus to be expected that the state corresponding to the level $k=1$ affine Lie algebra $\widehat{\mathrm{so}}(5)$ is related to the MR state. This is indeed the case; the wave function also contains a pfaffian factor [2]

$$
\widetilde{\Psi}_{\mathrm{SCsep}}^{k=1}=\operatorname{Pf}\left(\frac{1}{x_{i}-x_{j}}\right) \widetilde{\Psi}_{\mathrm{H}}^{M+1, M+1, M}\left(z_{i}^{\uparrow}, z_{j}^{\downarrow}\right)
$$

where $\widetilde{\Psi}_{\mathrm{H}}^{M+1, M+1, M}$ is the Halperin wave function (2). For $M$ odd, this describes a $\mathrm{qH}$ system at filling $\nu=\frac{2}{2 M+1}$. This wave function is different from the previous ones in a few respects. First of all, the (fundamental) excitations over this state show a separation of the spin and charge degrees of freedom (SCsep). This is a consequence of the structure of the underlying Lie algebra symmetry, as was pointed out in [2]. In effect, there are spinon like excitations, with spin- $\frac{1}{2}$ and no charge, and holons which carry charge $\frac{1}{2 M+1}$ without spin.

The other feature which is different in comparison to the clustered states (7) and (8) is the clustering property. The clustering of the 'spin charge separated state' (10) (with $M=0$ ), is in fact a clustering of the spin-up and spin-down particles separately. Note that the wave function for $M=0$ has poles when spin-up particles are at the same location as spin-down particles. Nevertheless, we will discuss the clustering for the case $M=0$. In the case $k=1$, up to two particles of the same spin can be brought to the same location while the wave function is still non-zero. If we first bring $z_{1}^{\uparrow}, z_{2}^{\uparrow} \rightarrow z^{\uparrow}$ and $z_{1}^{\downarrow}, z_{2}^{\downarrow} \rightarrow z^{\downarrow}$, and then send $z^{\uparrow} \rightarrow z^{\downarrow}$ the wave function remains non-zero. The clustering is thus different from the clustering of the NASS states (8), which vanish when any $k+1$ particles are brought together.

We refer to a forthcoming paper [3] for further analysis of the spincharge separated $\mathrm{qH}$ states for general $k$.

\section{Quasi-holes over paired and clustered qH states}

In a BCS superconductor, where electrons are paired up, the fundamental flux quantum is reduced to $\frac{1}{2} \Phi_{0}=\frac{h}{2 e}$. The same phenomenon occurs in the paired and clustered $\mathrm{qH}$ states, and this means that inserting a single flux quantum $\Phi_{0}$ creates more than a single quasi-hole. For the spin-polarized states of [24] the number of quasi-holes is given 
by $n=k \Delta N_{\phi}$, where $\Delta N_{\phi}$ is the number of excess flux quanta ${ }^{1}$. For the spin-singlet states of [5], this relation becomes $n^{\uparrow}+n^{\downarrow}=2 k \Delta N_{\phi}$, were $n^{\uparrow, \downarrow}$ denotes the number of spin-up and down quasi-holes, respectively.

The quasi-holes over the paired and clustered $\mathrm{qH}$ states carry fractional charge and satisfy non-abelian braid statistics. They can be studied with the help of the associated CFT. The wave functions of states in the presence of quasi-holes are obtained by inserting into the CFT correlators the appropriate quasi-hole operators, which consist of a vertex operator part and a spin field of the parafermion theory. In the case of the MR state, this is the spin field $\sigma$ of the Ising model and the quasi-hole operator becomes $\sigma(w): \exp \left(\frac{i}{2 \sqrt{M+1}} \varphi_{c}\right):(w)$.

The non-abelian statistics have their origin in the non-trivial fusion rules of the parafermion spin fields. In general, there is more than one way to fuse the fields in the correlator to the identity; for $n$ spin fields the number of ways will be denoted by $d_{n}$. The braiding of $n$ quasi-holes is then represented by a matrix of size $d_{n} \times d_{n}$.

\subsection{Braid statistics}

The simplest example that exhibits the non-abelian braiding is the situation where 4 quasi-holes are added to the MR state. In this case $d_{4}=2$, so there are two distinct states which, following [23], we write as $\Psi^{(4 \mathrm{qh}, 0)}$ and $\Psi^{\left(4 \mathrm{qh}, \frac{1}{2}\right)}$. Starting from the state $\Psi^{(4 \mathrm{qh}, 0)}$, and braiding two of the particles, we find the following transformation

$$
\Psi^{(4 \mathrm{qh}, 0)} \rightarrow \frac{e^{\frac{i \pi}{4}}}{\sqrt{2}}\left(\Psi^{(4 \mathrm{qh}, 0)}+\Psi^{\left(4 \mathrm{qh}, \frac{1}{2}\right)}\right)
$$

Wave functions for the MR state with $n$ quasi-holes can be written as $[24]$

$$
\begin{aligned}
& \widetilde{\Psi}_{\mathrm{MR}, \mathrm{qh}}\left(z_{1}, \ldots, z_{N} ; w_{1}, \ldots, w_{n}\right)=\frac{1}{2^{(N-F) / 2}(N-F) / 2 !} \prod_{i<j}\left(z_{i}-z_{j}\right)^{M+1} \\
& \times \sum_{\sigma \in S_{N}} \operatorname{sgn}(\sigma) \prod_{k=1}^{F} z_{\sigma(k)}^{m_{k}} \prod_{l=1}^{(N-F) / 2} \frac{\Phi\left(z_{\sigma(F+2 l-1)}, z_{\sigma(F+2 l)} ; w_{1}, \ldots, w_{n}\right)}{\left(z_{\sigma(F+2 l-1)}-z_{\sigma(F+2 l)}\right)}
\end{aligned}
$$


where

$$
\Phi\left(z_{1}, z_{2} ; w_{1}, \ldots, w_{n}\right)=\frac{1}{((n / 2) !)^{2}} \sum_{\tau \in S_{n}} \prod_{r=1}^{n / 2}\left(z_{1}-w_{\tau(2 r-1)}\right)\left(z_{2}-w_{\tau(2 r)}\right) .
$$

The integers $m_{1}, \ldots, m_{F}$ must be chosen such that they satisfy $0 \leq m_{1}<$ $m_{2}<\ldots<m_{F} \leq \frac{n}{2}-1$, giving rise to a degeneracy $d_{n}^{(F)}=\left(\begin{array}{c}\frac{n}{2} \\ F\end{array}\right)$. The number $F$ is interpreted as the number of unpaired electrons in the excited state.

The braid matrices for $n$ quasi-hole excitations were obtained by Nayak and Wilczek [23], who showed a direct connection with the rotation matrices of the group $S O(2 n)$. We refer to [27] for more general results on braid matrices.

\subsection{Quasi-hole counting formulas}

The CFT approach to the excited state wave functions and their braid properties is highly efficient. One would like however, to 'keep both feet on the ground' and understand the fundamental degeneracies that characterize the non-abelian statistics in a more direct way. This can be done by selecting a (ultra-local) hamiltonian that has the $\mathrm{qH}$ state as its ground state, and then (numerically) studying the spectrum of excited states.

These numerical computations are most easily performed by studying a small number of particles in a spherical geometry. By tuning the number of flux quanta to the value $N_{\phi}=\frac{1}{\nu} N-S$, where $S$ is the socalled shift [30], one realizes the $\mathrm{qH}$ state as the unique ground state. Cranking up the number $N_{\phi}$ and performing a numerical diagonalization, one obtains characteristic degeneracies for quasi-hole excitations.

Following [24], we first explain the counting of degeneracies for the case of the MR state. To understand the degeneracies of quasi-hole excitations on the sphere, two effects should be taken into account. The first is a choice of fusion path or, equivalently, a choice of numbers $F$ and $m_{1}, \ldots, m_{F}$ in the formula (11). The second effect is the socalled orbital degeneracy: the quasi-holes are not localized on the sphere, but can occupy one of a finite number of available orbitals, each of which is characterized by a definite angular momentum $L_{z}$. These orbital degeneracies are well-known from the analysis of integer and abelian $\mathrm{qH}$ states.

For the MR state, the orbital degeneracy factor depends on the number $F$ of unpaired electrons. Fixing this number $F$, we have $d_{n}^{(F)}$ different choices for the quasi-hole wave function. To each of those we can asso- 
ciate [24] an orbital degeneracy factor equal to $\left(\begin{array}{c}\frac{N-F}{2}+n \\ n\end{array}\right)$. Putting it all together, we have the following total degeneracy

$$
\#(N, n)=\sum_{F}\left(\begin{array}{c}
\frac{n}{2} \\
F
\end{array}\right)\left(\begin{array}{c}
\frac{N-F}{2}+n \\
n
\end{array}\right),
$$

in agreement with numerical results [24]. The degeneracies $d_{n}$ relevant for a situation where $n$ quasi-holes are at fixed positions are recovered by suppressing the orbital factors, $d_{n}=\sum_{F}^{\prime} d_{n}^{(F)}=2^{n / 2-1}$, where the sum is over even (odd) $F$ for $N$ even (odd). This number is in agreement with a direct count of the number of fusion paths of $n$ Ising spin fields [23].

For the more general clustered $\mathrm{qH}$ states, the degeneracies have basically the same form: an orbital part and an intrinsic part, stemming from the non-trivial fusion rules. The difference is however, that we can not rely on explicit wave functions to handle the intrinsic degeneracy. One can work around this by extracting from the parafermion CFT the relevant combinatorial factors, using the methods put forward in [26, 8].

For the RR states, the counting has been worked out in [15], with the result

$$
\#(N, n ; k)=\sum_{F}\left\{\begin{array}{l}
n \\
F
\end{array}\right\}_{k}\left(\begin{array}{c}
\frac{N-F}{k}+n \\
n
\end{array}\right),
$$

with $n$ the number of quasi-holes, $n=k \Delta N_{\phi}$. The symbols $\left\{\begin{array}{l}n \\ F\end{array}\right\}_{k}$ represent the degeneracies due to the fusion rules. In $[8,15]$, these were described in terms of recursion relations; explicit formulas (based on binomials) for general $k$ can be found in [6]. The sum $d_{n}=\sum_{F}\left\{\begin{array}{c}n \\ F\end{array}\right\}_{k}$, which equals the total number of fusion paths for the spin fields contained in $n$ quasi-hole operators, sets the dimension of the braid matrices for braiding 2 out of the $n$ quasi-particles.

For the NASS states of [5], the counting goes along the same lines, with the additional complication that we have to deal with two spin components, which are combined in a non-trivial way. This is reflected in the counting formulas by a doubling of the number of binomial factors. By inserting an amount $\Delta N_{\phi}$ of extra flux, one creates quasi-holes, which can have either spin. The total number of quasi-holes is fixed, $n^{\uparrow}+n^{\downarrow}=$ $2 k \Delta N_{\phi}$. The symbols \{\}$_{k}$ now depend on four parameters $\left\{\begin{array}{ll}n^{\uparrow} & n^{\downarrow} \\ F_{1} & F_{2}\end{array}\right\}_{k}$ and we have $d_{n^{\uparrow}, n \downarrow}=\sum_{F_{1}, F_{2}}\left\{\begin{array}{l}n^{\uparrow} \\ F_{1} \\ F_{2}\end{array}\right\}_{k}$. The case $k=2$ has been worked out in detail in [4], where the results are checked against numerical data. Explicit results for the symbols \{\}$_{k}$ can be found in [6]. The counting of quasi-hole degeneracies over the spin-charge separated spin-singlet states will be discussed in [3]. 
The numbers $d_{n}$ (for both spin-polarized and spin-singlet states) are easily extracted from the known fusion rules of the $\widehat{s u}(2)_{k}$ and $\widehat{s u}(3)_{k}$ CFTs. For both $\widehat{s u}(2)_{3}$ and $\widehat{s u}(3)_{2}$ the numbers $d_{n}$ are Fibonacci numbers. The asymptotic behavior for $n \rightarrow \infty$ is found to be

$$
d_{n} \sim\left[2 \cos \frac{\pi}{k+2}\right]^{n}
$$

for the RR states, and

$$
d_{p} \sim\left[1+2 \cos \frac{2 \pi}{k+3}\right]^{p}
$$

for the NASS states, where $p=n^{\uparrow}+n^{\downarrow}$.

\subsection{Exclusion statistics and $K$ matrix structure}

In [1] a proposal was made for a $K$-matrix structure of the paired and clustered $\mathrm{qH}$ states discussed in this paper. It was established that the exclusion statistics of edge excitations over these states (in a suitable basis) can be captured by a statistics matrix of the form (4), supplemented by a prescription that some of the particles described by this matrix be viewed as pseudo-particles. We refer to the first paper of [1] for a physical picture underlying these $K$-matrices, and to the second paper of [1] for mathematical details.

\section{Acknowledgments}

K.S. thanks A. Cappelli and G. Mussardo for putting together a most inspiring Workshop. We acknowledge collaboration with A.W.W. Ludwig, N. Read, E.H. Rezayi, and stimulating discussions with A. Cappelli and I.T. Todorov. This research is supported in part by the Foundation FOM of the Netherlands and by the Netherlands Organisation for Scientific Research (NWO).

\section{Notes}

1. Note that we adopt a slightly different notation than the one used in $[24,15]$. Here, $n$ denotes the number of quasi-holes, rather than the number of excess flux quanta.

\section{References}

[1] E. Ardonne, P. Bouwknegt, S. Guruswamy and K. Schoutens, Phys. Rev. B61, 10298 (2000); E. Ardonne, P. Bouwknegt and K. Schoutens, J. Stat. Phys. 102, 421 (2001). 
[2] E. Ardonne, F.J.M. van Lankvelt, A.W.W. Ludwig, and K. Schoutens, Phys. Rev. B, accepted for publication, [cond-mat/0102072].

[3] E. Ardonne, F.J.M. van Lankvelt, and K. Schoutens, paper in preparation.

[4] E. Ardonne, N. Read, E. Rezayi, and K. Schoutens, Nucl. Phys. B607, 549 (2001).

[5] E. Ardonne and K. Schoutens, Phys. Rev. Lett. 82, 5096 (1999).

[6] E. Ardonne, J. Phys. A, accepted for publication, [cond-mat/0110108].

[7] D. Arovas, J. R. Schrieffer and F. Wilczek, Phys. Rev. Lett. 53, 722 (1984).

[8] P. Bouwknegt and K. Schoutens, Nucl. Phys. B547, 501 (1999).

[9] A. Cappelli, L.S. Georgiev, and I.T. Todorov, Nucl. Phys. B599, 499 (2001).

[10] N. R. Cooper, N. K. Wilkin and J. M .F. Gunn, Phys. Rev. Lett. 87, 405 (2001).

[11] R. A. J. van Elburg and K. Schoutens, Phys. Rev. B58, 15704 (1998).

[12] D. Gepner, Nucl. Phys. B290 10 (1987).

[13] G. A. Goldin, R. Menikoff and D. H. Sharp, Phys. Rev. Lett. 54, 603 (1985).

[14] M. Greiter, X.-G. Wen and F. Wilczek, Nucl. Phys. B374, 567 (1992).

[15] V. Gurarie and E. Rezayi, Phys. Rev. B61, 5473 (2000).

[16] F. D. M. Haldane, Phys. Rev. Lett. 67, 937 (1991).

[17] B. I. Halperin, Phys. Rev. Lett. 52, 1583 (1984).

[18] B. Halperin, Helv. Phys. Acta 56, 75 (1983).

[19] S. B. Isakov, G. S. Canright and M. D. Johnson, Phys. Rev. B55, 6727 (1997).

[20] R. B. Laughlin, Phys. Rev. Lett. 50, 1395 (1983).

[21] R. H. Morf, Phys. Rev. Lett. 80, 1505 (1998); E. Rezayi and F. D. M. Haldane, Phys. Rev. Lett. 84, 4685 (2000).

[22] G. Moore and N. Read, Nucl. Phys. B360, 362 (1991).

[23] C. Nayak and F. Wilczek, Nucl. Phys. B479, 529 (1996).

[24] N. Read and E. Rezayi, Phys. Rev. B54, 16864 (1996).

[25] N. Read and E. Rezayi, Phys. Rev. B59, 8084 (1999).

[26] K. Schoutens, Phys. Rev. Lett. 79, 2608 (1997).

[27] J.K. Slingerland and F.A. Bais, Nucl. Phys. B612, 229 (2001).

[28] D. C. Tsui, H. L. Störmer and A. C. Gossard, Phys. Rev. Lett. 48, 1559 (1982).

[29] X.-G. Wen, Phys. Rev. B41, 12838 (1990); Int. Jour. Mod. Phys. B6, 1711 (1992).

[30] X.-G. Wen, Adv. Phys. 44, 405 (1995).

[31] X.-G. Wen, Phys. Rev. Lett. 66, 802 (1991); B. Blok and X.-G. Wen, Nucl. Phys. B374, 615 (1992); X.-G. Wen, cond-mat/9811111; X.-G. Wen and A. Zee, Phys. Rev. B58, 15717 (1998).

[32] R. E. Willett et al., Phys. Rev. Lett. 59, 1776 (1987); W. Pan et al., Phys. Rev. Lett. 83, 3530 (1999).

[33] E. Witten, Comm. Math. Phys. 121, 351 (1989).

[34] A. B. Zamolodchikov and V. A. Fateev, Sov. Phys. JETP 62, 215 (1985). 